\title{
Formulation and Characterization of Hydroquinone Nanostructured Lipid Carriers by Homogenization Emulsification Method
}

\author{
Pey-Shiuan Wu, ${ }^{1}$ Chih-Hung Lin, ${ }^{1}$ Yi-Ching Kuo, ${ }^{1,2}$ and Chih-Chien Lin ${ }^{1}$ \\ ${ }^{1}$ Department of Cosmetic Science, Providence University, Taichung, Taiwan \\ ${ }^{2}$ Department of Applied Chemistry, Providence University, Taichung, Taiwan \\ Correspondence should be addressed to Chih-Chien Lin; chchlin@pu.edu.tw
}

Received 5 January 2017; Revised 24 April 2017; Accepted 4 May 2017; Published 1 June 2017

Academic Editor: Takuya Tsuzuki

Copyright (C) 2017 Pey-Shiuan Wu et al. This is an open access article distributed under the Creative Commons Attribution License, which permits unrestricted use, distribution, and reproduction in any medium, provided the original work is properly cited.

Nanostructured lipid carrier (NLC) was prepared by homogenization emulsification method and improved with modified surfactants. The properties of nanoparticles were investigated using the NLC system for hydroquinone (HQ) as a model drug and by increasing the light stability of hydroquinone. The optimized condition of NLC in stirring was $1200 \mathrm{rpm}$, the homogenized speed was $8000 \mathrm{rpm}$, solid oil to liquid oil ratio was $3: 7$, and lecithin to surfactant ratio was $3: 1$. The particle size was $393.30 \pm 28.23 \mathrm{~nm}$ and the encapsulation efficiency was $22.13 \pm 2.66 \%$. The zeta-potential of HQ-NLC was better than $-30 \mathrm{mV}$. In the thermogravimetric analysis (TGA) studies, adding of PLANTACARE 2000 UP for HQ-NLC has better heat resisting property than the HQNLC only. The addition of PLANTACARE 2000 UP to NLC shows better permeability (125.10\%) than that of Blank. In the stability studies, the HQ-NLC after UVA/UVB irradiation has better inhibition rate (34.25\%) than that of the Blank. In the present study, NLC system has successfully improved the light stability and the skin permeability of active compound. Therefore, the NLC might be a potential delivery vehicle for transdermal products in the future.

\section{Introduction}

Hydroquinone (HQ) is a pharmaceutical compound used not only as a skin-whitening agent, but also to condense tyrosinase in the generation mechanism of melanin $[1,2]$. Hydroquinone can prevent tyrosine, dopa, and dopaquinone after a series of reaction of melanin and may achieve the whitening effect. Hydroquinone is often used to treat lentigines [3], freckle, ephelides, pigmentation after inflammation (postinflammatory hyperpigmentation) [4], liver spots (chloasma, melasma), and other diseases. It may cause skin irritation with many adverse effects. Excessive amounts of HQ can kill the melanoblast and cause skin disease called Vitiligo $[5,6]$.

Nanostructured lipid carrier (NLC) is the evolution for solid lipid nanoparticles (SLN) [7], with some improvements in the encapsulation efficiency. The encapsulation efficiency of NLC is better than the encapsulation efficiency of SLN because solid-liquid mixing proportion is better than single solid lipid. Although both have the preparation methods, NLC may disrupt the crystal structure of regular solid lipid. When liquid lipid is added, it may cause irregular particle structure, increase the ratio of crystalline, and thus increase the encapsulation efficiency and drug loading $[8,9]$. The NLC system not only increases the stability of the compound, but also has good skin adhesion and bioavailability. When the particle adheres to the skin surface, it will accumulate to generate packets action effect, which reduces water loss in the skin surface and increases skin hydration in order to protect the skin.

Nanoparticles may be biodegradable and not harmful to organisms in the environment. However, hydroquinone rapidly oxidizes in sunlight and loses its active effect [10]. In this study, we used homogenization emulsification method to prepare the HQ-NLC [11]. We found that this technology of delivery system can improve photosensitivity of hydroquinone in UV light and also enhance the stability of the 
product and then achieve great drug delivery and reduce skin irritation in human body.

\section{Materials and Methods}

2.1. Materials. Hydroquinone (HQ) was purchased from Sigma. Bees wax, Span 80 (sorbitan monooleate), and Tegosoft CT (caprylic/capric triglyceride) were purchased from LUBRIZOL. Lecithin was purchased from LucasMeyer. Sodium hydrogen sulfite was purchased from Sigma. PLANTACARE 2000 UP (INCI: decyl glucoside, C8-C16 fatty alcohol polyglycoside) was purchased from BASF. Ethanol, methanol (HPLC grade), and all other chemicals were obtained from Acros Organics. Artificial skin membrane (Tegaderm $^{\mathrm{TM}}$, 90022TCP) was purchased from 3M Company.

2.2. Preparation for Optimized Condition of HQ-NLC. The solution, $5.0 \%$ hydroquinone, $0.1 \%$ sodium hydrogen sulfite, and $75.4 \% \mathrm{DD}$-water in the aqueous phase, was heated at $80^{\circ} \mathrm{C}$. At the same time, mixed $4.5 \%$ bees wax, $10.5 \%$ caprylic/capric triglyceride (CCTG), 3\% lecithin, and 1.5\% Span 80 in the lipid phase were heated at $80^{\circ} \mathrm{C}$. The water phase was added to a hot-melt lipid phase. The mixture was stirred at 600 1200 rpm for $20 \mathrm{~min}$ in ultrasonication [12] and then homogenized at 4000 10000 rpm for $10 \mathrm{~min}$. After a process of preparation, remove solvents in sample by Rotary Vacuum Evaporator at $60^{\circ} \mathrm{C}$. We used the optimized condition to prepare the NLC before adding the modified surfactant. The oil phase was mixed with $4.5 \%$ bees wax, $10.5 \%$ caprylic/capric triglyceride (CCTG), 3\% lecithin, and 1.5\% PLANTACARE 2000 UP. There were two kinds of lecithin ratio: PLANTACARE 2000 UP to use about $2: 1$ and $1: 2$.

2.3. Measurement of Particle Size and Zeta-Potential. The measuring range of Zetasizer Nano-ZS is approximately from $0.6 \mathrm{~nm}$ to $6 \mu \mathrm{m}$. The NLC samples were diluted with $10 \%$ Sodium Lauryl Ether Sulphate (SLES) to detect the particle size and polydispersity index (PdI). The NLC samples were diluted with DD-water to detect the zeta-potential by Laser Particle Size Analyzer/MALVERN Nano-ZS. Before testing, the NLC samples were suspended by ultrasonication.

2.4. The Determination of Encapsulation Efficiency. High-performance liquid chromatography system (pump: PU/1580, Jasco) with reversed-phase C18 column $(250 \mathrm{~mm} \times 4.6 \mathrm{~mm}$, pore is $5 \mu \mathrm{m}$, Agilent) was used to detect hydroquinone. The composition of mobile phase was $40 \%$ methanol and $60 \%$ phosphate buffer solution $(0.02 \mathrm{M}, \mathrm{pH}=5.5)$ [13]; the flow-rate was $0.8 \mathrm{~mL} / \mathrm{min}$. The injection of sample solution was $20 \mu \mathrm{L}$. The UV detector was set at $280 \mathrm{~nm}$ to detect hydroquinone. All experiments were performed in triplicate. The encapsulation efficiency (E.E.\%) of hydroquinone in the NLC was determined indirectly by HPLC method; the free amounts of HQ in solution were separated by filtration method and then determined by HPLC. The encapsulation efficiency and the drug loading (D.L.\%) were then calculated via

$$
\begin{aligned}
& \text { E.E. }(\%) \\
& =\frac{\text { total amount of HQ }- \text { free amount of HQ }}{\text { total amount of HQ }} \\
& \quad \times 100 \%, \\
& \text { D.L. }(\%)=\frac{\text { weight of HQ in NLC }}{\text { weight of NLC }} \times 100 \% .
\end{aligned}
$$

2.5. The Thermogravimetric Analysis. Thermogravimetric analysis (TGA) instrument was used on Perkin Elmer Pyris 1 TGA instrument. For a normal experiment, 5-10 mg HQNLC was put in an alumina crucible. Then, the samples were heated in nitrogen gas flow at a rate of $10^{\circ} \mathrm{C} / \mathrm{min}$ from $25^{\circ} \mathrm{C}$ to $600^{\circ} \mathrm{C}$.

2.6. The Scanning Electron Microscopy Analysis. Scanning electron microscopy (SEM) was used on a JEOL JSM-6700F instrument operating at $15 \mathrm{kV}$. For SEM characterization, the HQ-NLC was added to the copper grid coated with carbon film. Before observations of SEM, all samples were heated at $60^{\circ} \mathrm{C}$ to dry and coated with Platinum onto HQ-load NLCs for about $70 \mathrm{~s}$ in $20 \mathrm{~mA}$.

2.7. In Vitro Skin Permeation Study. The in vitro skin permeation study with hydroquinone-load nanostructured lipid carrier used a Franz-type diffusion cell system. In the study, we used artificial skin membranes (3M Tegaderm) to simulate the stratum corneum side of human skin. The surface area of the release membrane was $0.785 \mathrm{~cm}^{2}$ and the volume of the receptor phase was approximately $5 \mathrm{ml}$. The receptor cell was filled with phosphate buffer solution $(0.155 \mathrm{M}, \mathrm{pH}$ 7.0 ); then $1 \mathrm{ml}$ of each sample (5\% HQ) was added to the donor cell. The stirring rate and temperature were kept at $300 \mathrm{rpm}$ and $36.7 \pm 0.3^{\circ} \mathrm{C}$. Experimental time was $0.5,1,2$, 4 , and $6 \mathrm{~h}$. The experiments were performed in triplicate and the concentration of hydroquinone in receptor media was detected by HPLC.

2.8. Inhibition Tyrosinase Assay with UVA/UVB Irradiation. The phosphate buffer ( $\mathrm{pH}$ 6.5) was added to $7 \%$ tyrosine solutions and the different concentrations of sample solution (hydroquinone solutions, HQ-load NLC solutions) before heating at $37^{\circ} \mathrm{C}$ in water-bath for $10 \mathrm{~min}$; then tyrosinase (100 units $/ \mathrm{mL}$ ) was added to PBS buffer and DD-water before heating at $37^{\circ} \mathrm{C}$ for $20 \mathrm{~min}$. Hydroquinone and HQload NLC were compared to expose the UVA/UVB with $17.5 \mathrm{~J} / \mathrm{cm}^{2}$ energy irradiation [14]. The results were detected using ELISA-reader scan at $475 \mathrm{~nm}$ to calculate the inhibition tyrosinase rate.

2.9. Statistical Analysis. The quantitative data for the present study were analyzed using Student's $t$-tests and are presented as the means \pm S.E. for three independent experiments. 


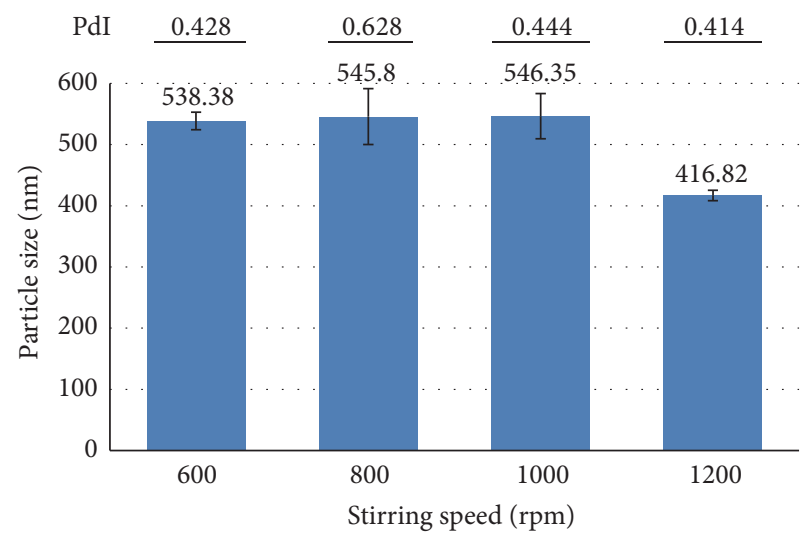

(a)

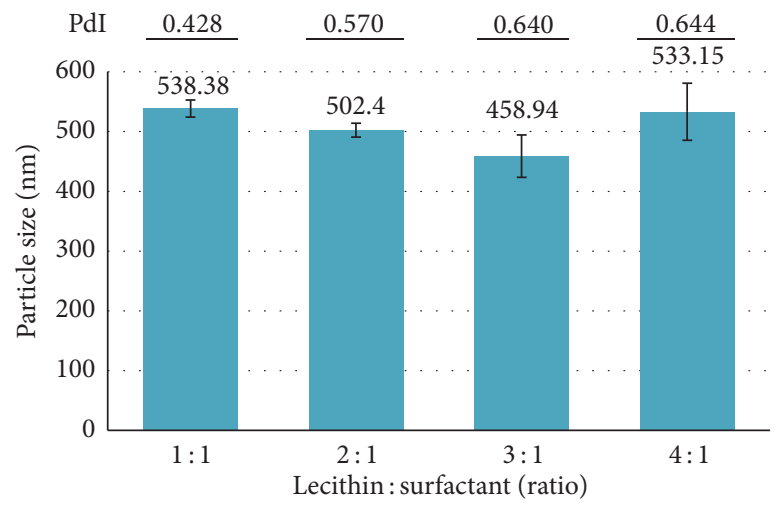

(c)

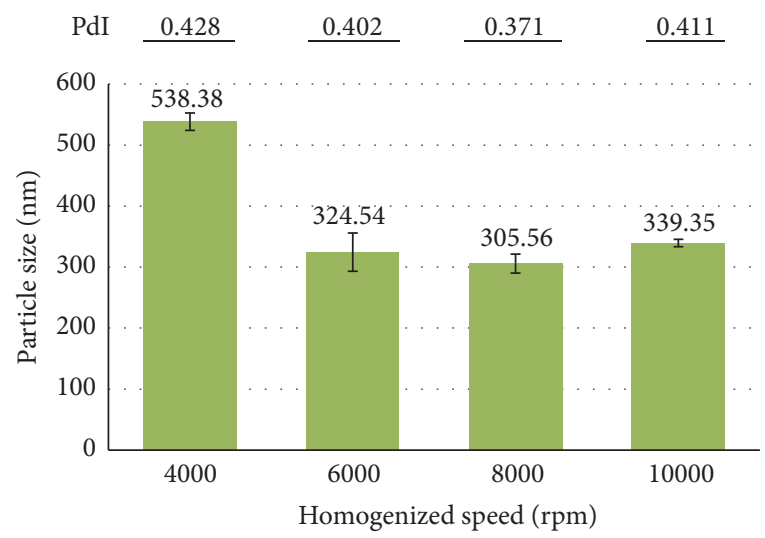

(b)

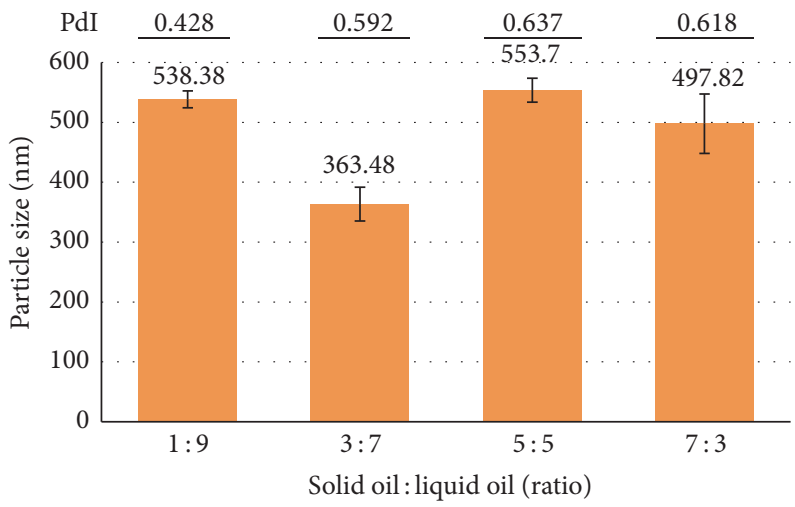

(d)

FIGURE 1: The particle size and PdI of NLC in different preparation conditions. (a) Stirring speed, (b) homogenized speed, (c) lecithin and surfactant ratio, and (d) solid oil and liquid oil ratio.

\section{Results and Discussion}

3.1. The Optimal of NLC Preparation. In the presented study, we used homogenization emulsification method to make the HQ-NLC. After all preparation processes, the activity of captured HQ was firstly checked by tyrosinase inhibition assay. The results demonstrated that HQ can retain its activity after all the preparation processes including high temperature condition (data not show).

In Figure 1, we investigated the conditions to prepare HQ-NLC, and there are 4 different stirring speeds used in this experiment: $600 \mathrm{rpm}, 800 \mathrm{rpm}, 1000 \mathrm{rpm}$, and $1200 \mathrm{rpm}$. The best stirring speed is $1200 \mathrm{rpm}$, the smallest particle diameter under this speed is $421.63 \pm 8.64 \mathrm{~nm}$. Due to high agitation speed, it can make HQ-NLC evenly dispersed and reduce agglomeration when preparing with a finer end result [15-17]. For homogenization, the particles are homogenized at 4 different speeds $(4000 \mathrm{rpm}, 6000 \mathrm{rpm}, 8000 \mathrm{rpm}$, and $10000 \mathrm{rpm}$ ) in this experiment. We get the best result when centrifuged at $8000 \mathrm{rpm}$, and the smallest particle diameter under this speed is $305.56 \pm 15.69 \mathrm{~nm}$. According to other studies, when stirring at higher speeds, smaller particle size is found due to high shear force that destroys the particle and causes cohesion $[18,19]$. The ratios of the solid oil and liquid oil are $1: 9,3: 7,5: 5$, and $7: 3$. When the ratio of the solid oil and liquid oil is $3: 7$, HQ-NLC could have the smallest particle size $(363.48 \pm 28.23 \mathrm{~nm})$. There is a literature that reported the enhancing ratio of the solid oil found in small particle size, but increase in the percentage of solid lipid generated particle agglomeration effect that leads to increased particle size [20]. Besides, lecithin and emulsifier ratios are $1: 1,2: 1,3: 1$, and $4: 1$, and the ratio of $3: 1$ enables HQ-NLC to have a minimum particle size of $458.94 \pm 35.36 \mathrm{~nm}$. The higher the percentage of lecithin, the lower the percentage of surfactant. Therefore, the ratio of $4: 1$ may cause NLC to be unstable. There is an article to point out that lecithin is a kind of emulsifier helper (cosurfactant), but more lecithin may cause particle agglomeration [21]; and at a lower percentage of emulsifier addition, the formulation of NLC may be unstable and the particles may be easy to gather due to larger diameter. PLANTACARE 2000 UP is the decyl glucoside based liquid type emulsifier. We established that PLANTACARE 2000 UP is a suitable main surfactant to combine lecithin for the production of NLC because of its medium size of structure.

In this study, we found the best of the preparation conditions for stirring speed to be $1200 \mathrm{rpm}$, the homogeneous speed to be $8000 \mathrm{rpm}$, the solid oil and liquid oil ratio to be $3: 7$, and the ratio of lecithin and emulsifier to 


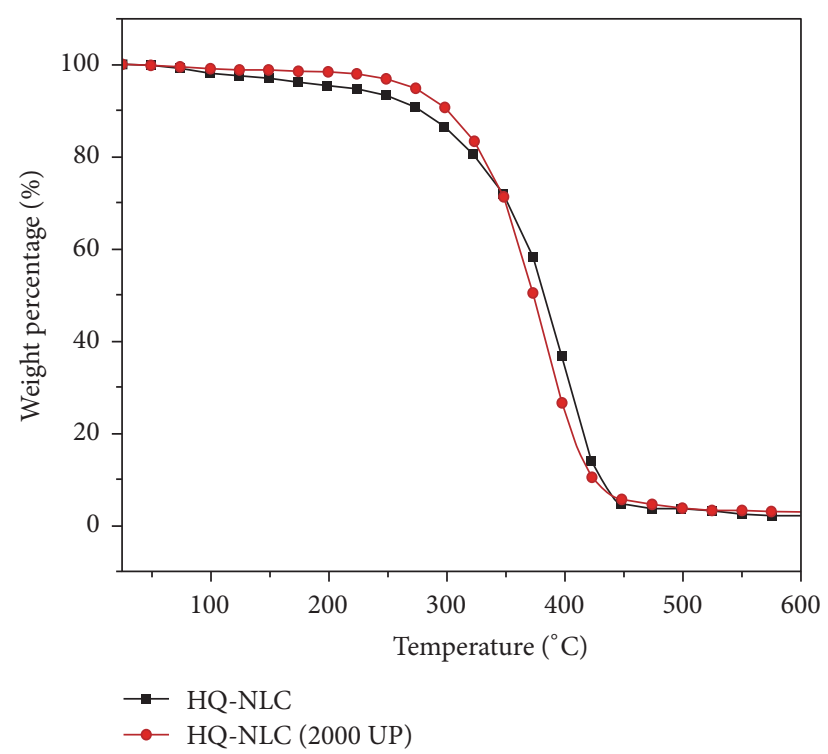

Figure 2: The thermogravimetric analyzer of HQ-NLC and HQNLC with PLANTACARE 2000 UP.

be $3: 1$; we found the smallest particle size of NLC to be $393.30 \pm 28.23 \mathrm{~nm}$. After adding modified emulsifier known as $2000 \mathrm{UP}$, the particle sizes of HQ-2000UP-NLC in ratios $2: 1$ and $1: 2$ are $396.60 \pm 27.39 \mathrm{~nm}$ and $461.34 \pm 18.14 \mathrm{~nm}$, respectively. Besides, the PdI of HQ-2000UP-NLC in ratios $2: 1$ and $1: 2$ are 0.583 and 0.563 , respectively.

3.2. The Zeta-Potential of NLC Analysis. In this study, the surface charge of the HQ-NLC is $-15.93 \pm 2.02 \mathrm{mV}$. The charges of HQ-2000UP-NLC in $2: 1$ ratio is $-34.86 \pm 2.54 \mathrm{mV}$ and the charge in $1: 2$ ratio is $-39.22 \pm 1.43 \mathrm{mV}$. When we add more proportion of modified emulsifiers with 2000 UP, the charge amount of NLC surface also increases. Some parts of the literature indicate that the higher zeta-potential is the less prone it is to agglomeration and it can increase stability during storage. Therefore, we select the modified emulsifier which causes zeta-potential more than $-30 \mathrm{mV}$ because the surface charge more than $\pm 30 \mathrm{mV}$ may have good particle stability and the zeta-potential of NLC greater than $\pm 60 \mathrm{mV}$ may have excellent stability [22]. The modified emulsifier PLANTACARE 2000 UP is composed of C8-C16 fatty alcohol polyglycoside. Supposedly, the glycoside part may offer the higher surface charge in NLC.

3.3. Encapsulation Efficiency Analysis. There are three kinds of NLC in this study: HQ-NLC and HQ-2000UP-NLC with two ratios, $2: 1$ and $1: 2$, respectively. Their encapsulation efficiencies were $19.52 \% \pm 2.51 \%, 22.13 \% \pm 2.66 \%$, and $26.84 \% \pm 1.20 \%$. We chose the HQ-2000UP-NLC with ratio $2: 1$ since it has the highest drug loading $(19.28 \pm 4.77 \%)$.

3.4. The Thermogravimetric Analysis. In Figure 2, the samples were not heated prior to percentages by weight (100\%), and after the start of heating, it will continue to reach the weight loss after pyrolysis. In order to verify the conformation of carbon black, condition of gas was converted into oxygen, and the extent of the weight loss period interval can be suggested as carbon black. In this figure, HQ-2000UP-NLC reveals a higher heat resistance than HQ-NLC because, during TGA heating process, HQ-2000UP-NLC has a lower pyrolysis temperature (the temperature for $95 \%$ of cumulative percentage of weight) than that of HQ-NLC $[23,24]$. The decomposition temperature (abbreviated Td5) was $271.55 \pm$ $0.17^{\circ} \mathrm{C}$, higher than the cracking temperature of HQ-NLC which is $215.77 \pm 0.16^{\circ} \mathrm{C}$. In addition, we can observe that the temperature reaches $450^{\circ} \mathrm{C}$, either HQ-2000UP-NLC or HQ-NLC powder was burned out, and do not leave any coke because NLC powder is organic compounds composed of representation. The result not only shows the particles' biodegradable availability, but also refers to what may not cause the cumulative pollution to environment. The above experiments HQ-2000UP-NLC result values are greater than the HQ-NLC; therefore, the following are with HQ-2000UPNLC as the experimental group.

3.5. The Scanning Electron Microscopy Analysis. In Figure 3(a), we found that the HQ-NLC presents a sphere-like shape in the SEM image observation. In Figure 3(b), the SEM image observed in most of the HQ-2000UP-NLC has similar particle size as observed in the residue after the damaged part of the particles. From the SEM image observation in Figure 3, we noticed the broken parts of HQ-2000UP-NLC. In Figure 3(d) with SEM image observation, we found that there are many black shadows on HQ-2000UP-NLC shell material indicating spaces in the particles.

3.6. In Vitro Skin Permeation Study. In Figure 4, we found that the penetration ratio of HQ-2000UP-NLC is higher than the hydroquinone solution after $6 \mathrm{~h}$. Adding modified emulsifier called PLANTACARE 2000 UP caused the HQ-2000UP-NLC to have better penetration rate. The reason is that PLANTACARE 2000 UP emulsifier has more hydrophilic structure, which was a better stratum corneum hydration to increase the penetration of HQ-2000UPNLC. The experimental results showed that, at the second hour, the percutaneous penetration of HQ-2000UP-NLC is $0.285 \pm 0.003 \%$, which was significantly greater than that of HQ $(0.126 \% \pm 0.016 \%)$.

In this study, the artificial skin membrane was used to simulate the stratum corneum side of human skin. For the in vitro skin permeation study, an artificial skin membrane provides a human skin-like structure and thickness, which is quite stable for quality than that of human and porcine skin sample. Therefore, our results demonstrated that the percutaneous penetration ability of HQ-2000UP-NLC was significantly greater than HQ only group. Theoretically, the consequence should be the same with that of an in vivo study.

3.7. Inhibition Tyrosinase Assay with UVA/UVB Irradiation. In Figure 5, we used the same concentration of samples to test. The tyrosinase inhibition rate of hydroquinone solution was $53.91 \% \pm 1.78 \%$ and the tyrosinase inhibition rate of HQ-2000UP-NLC was $48.77 \% \pm 0.93 \%$. After $17.5 \mathrm{~J} / \mathrm{cm}^{2}$ of 


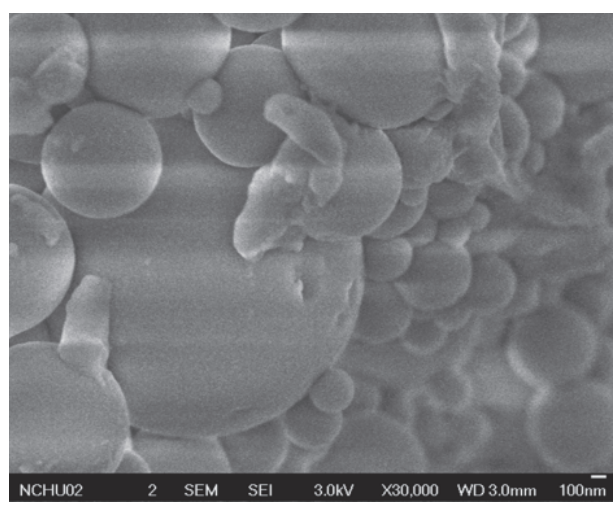

(a)

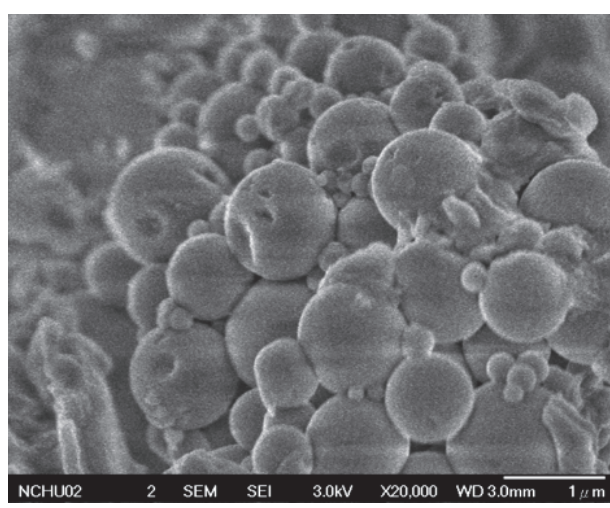

(c)

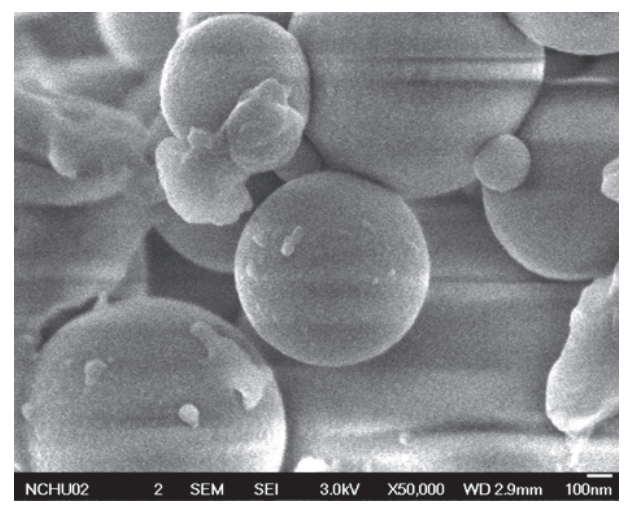

(b)

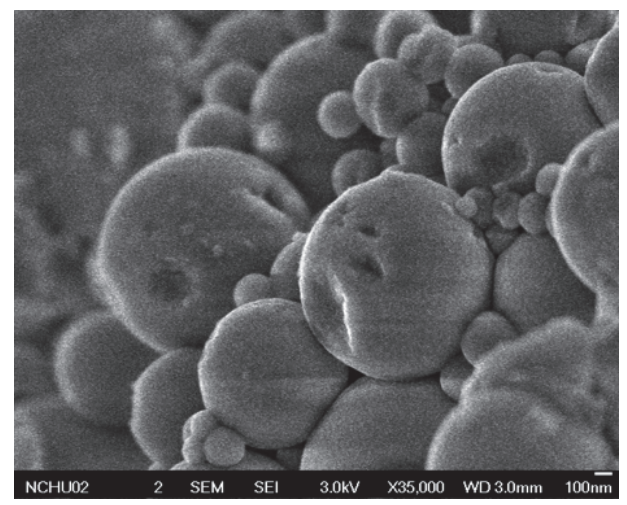

(d)

Figure 3: The SEM image of HQ-NLC (a), HQ-2000UP-NLC (b), HQ-2000UP NLC broken form (c), and zoom in HQ-2000UP-NLC broken form (d).

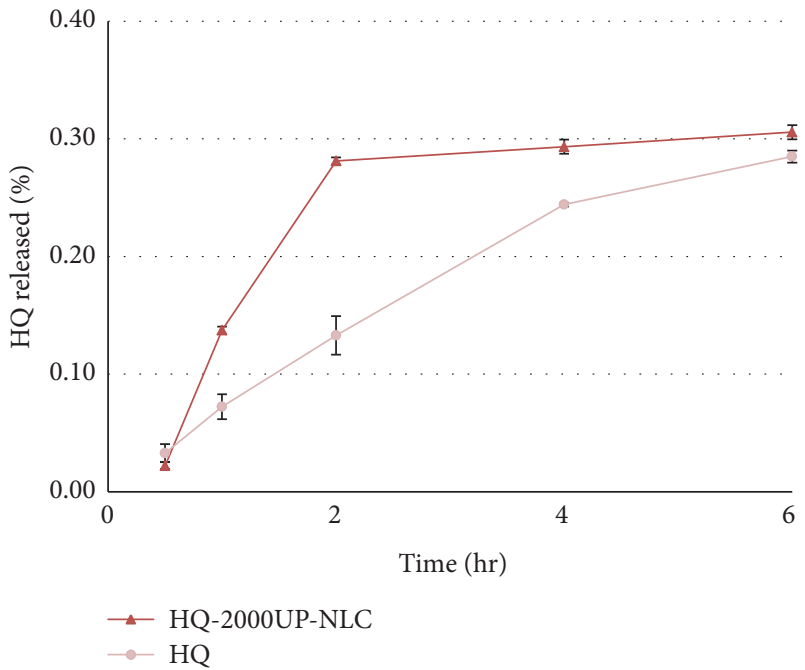

FIGURE 4: In vitro release of NLC with PLANTACARE 2000 UP.

UV energy irradiation (UVA, UVB) [25], the tyrosinase inhibition rate of HQ was $28.40 \% \pm 1.12 \%$ compared to the previous original inhibition rate of HQ when it decreases by $47.33 \%$. In the same way, the tyrosinase inhibition rate

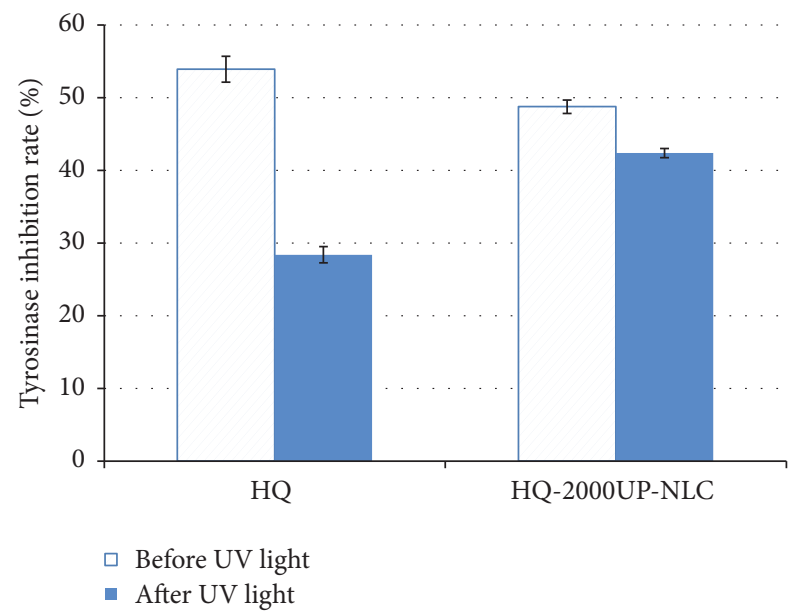

FIGURE 5: Light stability studies with HQ and HQ-2000UP-NLC.

of HQ-2000UP-NLC was $42.39 \% \pm 0.63 \%$ compared to the previous original inhibition rate of HQ-NLC when it decreases by $13.08 \%$. In the study, we proved that NLC system can effectively protect hydroquinone in order to reduce the 
damage of the UV light and that HQ-NLC also has a good value when applied in the product.

\section{Conclusion}

In summary, we established the best conditions for preparation of HQ-NLC. After the adding of PLANTACARE 2000 UP, there was no significant difference in particle size, but increase in the E.E. and D.L. in HQ-2000UP-NLC. In addition, all features of HQ-2000UP-NLC including zetapotential, thermal stability, in vitro permeability, and UV light resistance are better than that of HQ groups. Therefore, in the study, PLANTACARE 2000 UP based NLC system for HQ has successfully developed. Furthermore, the HQ2000UP-NLC might be a potential delivery vehicle for transdermal products in the future.

\section{Conflicts of Interest}

The authors declare that they have no conflicts of interest.

\section{Acknowledgments}

The Ministry of Science and Technology of Taiwan financially supported part of this work (104-2622-E-126-003-CC1, 1052622-E-126-001-CC2, and 105-2628-B-126-002-MY3).

\section{References}

[1] L.-H. Peng, S. Liu, S.-Y. Xu et al., "Inhibitory effects of salidroside and paeonol on tyrosinase activity and melanin synthesis in mouse B16F10 melanoma cells and ultraviolet B-induced pigmentation in guinea pig skin," Phytomedicine, vol. 20, no. 12, pp. 1082-1087, 2013.

[2] M. D. M. García-Molina, J. L. Muñoz Muñoz, F. Martinez-Ortiz et al., "Tyrosinase-catalyzed hydroxylation of hydroquinone, a depigmenting agent, to hydroxyhydroquinone: a kinetic study," Bioorganic and Medicinal Chemistry, vol. 22, no. 13, pp. 33603369, 2014.

[3] J.-P. Ortonne, A. G. Pandya, H. Lui, and D. Hexsel, "Treatment of solar lentigines," Journal of the American Academy of Dermatology, vol. 54, no. 5, pp. S262-S271, 2006.

[4] S. Briganti, E. Camera, and M. Picardo, "Chemical and instrumental approaches to treat hyperpigmentation," Pigment Cell Research, vol. 16, no. 2, pp. 101-110, 2003.

[5] Z.-M. Hu, Q. Zhou, T.-C. Lei, S.-F. Ding, and S.-Z. Xu, "Effects of hydroquinone and its glucoside derivatives on melanogenesis and antioxidation: biosafety as skin whitening agents," Journal of Dermatological Science, vol. 55, no. 3, pp. 179-184, 2009.

[6] S. Uddin, A. Rauf, T. G. Kazi, H. I. Afridi, and G. Lutfullah, "Highly sensitive spectrometric method for determination of hydroquinone in skin lightening creams: application in cosmetics," International Journal of Cosmetic Science, vol. 33, no. 2, pp. 132-137, 2011.

[7] M. Nassimi, C. Schleh, H.-D. Lauenstein et al., "Low cytotoxicity of solid lipid nanoparticles in in vitro and ex vivo lung models," Inhalation Toxicology, vol. 21, no. 1, pp. 104-109, 2009.

[8] R. H. Müller, S. Maassen, C. Schwarz, and W. Mehnert, "Solid lipid nanoparticles (SLN) as potential carrier for human use: interaction with human granulocytes," Journal of Controlled Release, vol. 47, no. 3, pp. 261-269, 1997.

[9] S. Ghanbarzadeh, R. Hariri, M. Kouhsoltani, J. Shokri, Y. Javadzadeh, and H. Hamishehkar, "Enhanced stability and dermal delivery of hydroquinone using solid lipid nanoparticles," Colloids and Surfaces B: Biointerfaces, vol. 136, pp. 1004-1010, 2015.

[10] R. H. Müller, M. Radtke, and S. A. Wissing, "Solid lipid nanoparticles (SLN) and nanostructured lipid carriers (NLC) in cosmetic and dermatological preparations," Advanced Drug Delivery Reviews, vol. 54, supplement 1, pp. S131-S155, 2002.

[11] C. H. Lin, A hydroquinone-loaded nanostructured lipid carrier for preparation, efficacy and stability, [M.S. thesis], Providence University, 2015.

[12] M. A. Schubert and C. C. Müller-Goymann, "Characterisation of surface-modified solid lipid nanoparticles (SLN): influence of lecithin and nonionic emulsifier," European Journal of Pharmaceutics and Biopharmaceutics, vol. 61, pp. 77-86, 2005.

[13] T. M. Göppert and R. H. Müller, "Protein adsorption patterns on poloxamer-and poloxamine-stabilized solid lipid nanoparticles (SLN)," European Journal of Pharmaceutics and Biopharmaceutics, vol. 60, no. 3, pp. 361-372, 2005.

[14] C.-H. Lin, J.-Y. Sheu, H.-L. Wu, and Y.-L. Huang, "Determination of hydroquinone in cosmetic emulsion using microdialysis sampling coupled with high-performance liquid chromatography," Journal of Pharmaceutical and Biomedical Analysis, vol. 38, no. 3, pp. 414-419, 2005.

[15] E. B. Souto and R. H. Müller, "Cosmetic features and applications of lipid nanoparticles (SLN, NLC)," International Journal of Cosmetic Science, vol. 30, no. 3, pp. 157-165, 2008.

[16] R. H. Müller, R. D. Petersen, A. Hommoss, and J. Pardeike, "Nanostructured lipid carriers (NLC) in cosmetic dermal products," Advanced Drug Delivery Reviews, vol. 59, no. 6, pp. 522530, 2007.

[17] W. Mehnert and K. Mäder, "Solid lipid nanoparticles: production, characterization and applications," Advanced Drug Delivery Reviews, vol. 47, no. 2-3, pp. 165-196, 2001.

[18] P. Ahlin, J. Kristl, and J. Šmid-Korbar, "Optimization of procedure parameters and physical stability of solid lipid nanoparticles in dispersions," Acta Pharmaceutica, vol. 48, no. 4, pp. 259267, 1998.

[19] R. H. Müller, K. Mäder, and S. Gohla, "Solid lipid nanoparticles (SLN) for controlled drug delivery-a review of the state of the art," European Journal of Pharmaceutics and Biopharmaceutics, vol. 50, no. 1, pp. 161-177, 2000.

[20] W. Mehnert and K. Mäder, "Solid lipid nanoparticles: production, characterization and applications," Advanced Drug Delivery Reviews, vol. 64, pp. 83-101, 2012.

[21] B. Siekmann and K. Westesen, "P234 solid lipid nanoparticles stabilized by tyloxapol," European Journal of Pharmaceutical Sciences, vol. 2, no. 1-2, p. 177, 1994.

[22] S. A. Wissing and R. H. Müller, "Solid lipid nanoparticles as carrier for sunscreens: in vitro release and in vivo skin penetration," Journal of Controlled Release, vol. 81, no. 3, pp. 225-233, 2002.

[23] J. E. Riviere and M. G. Papich, "Potential and problems of developing transdermal patches for veterinary applications," Advanced Drug Delivery Reviews, vol. 50, no. 3, pp. 175-203, 2001.

[24] S. Mukherjee, S. Ray, and R. S. Thakur, "Solid lipid nanoparticles: a modern formulation approach in drug delivery system," 
Indian Journal of Pharmaceutical Sciences, vol. 71, no. 4, pp. 349358, 2009.

[25] B. W. Barry, "Novel mechanisms and devices to enable successful transdermal drug delivery," European Journal of Pharmaceutical Sciences, vol. 14, no. 2, pp. 101-114, 2001. 

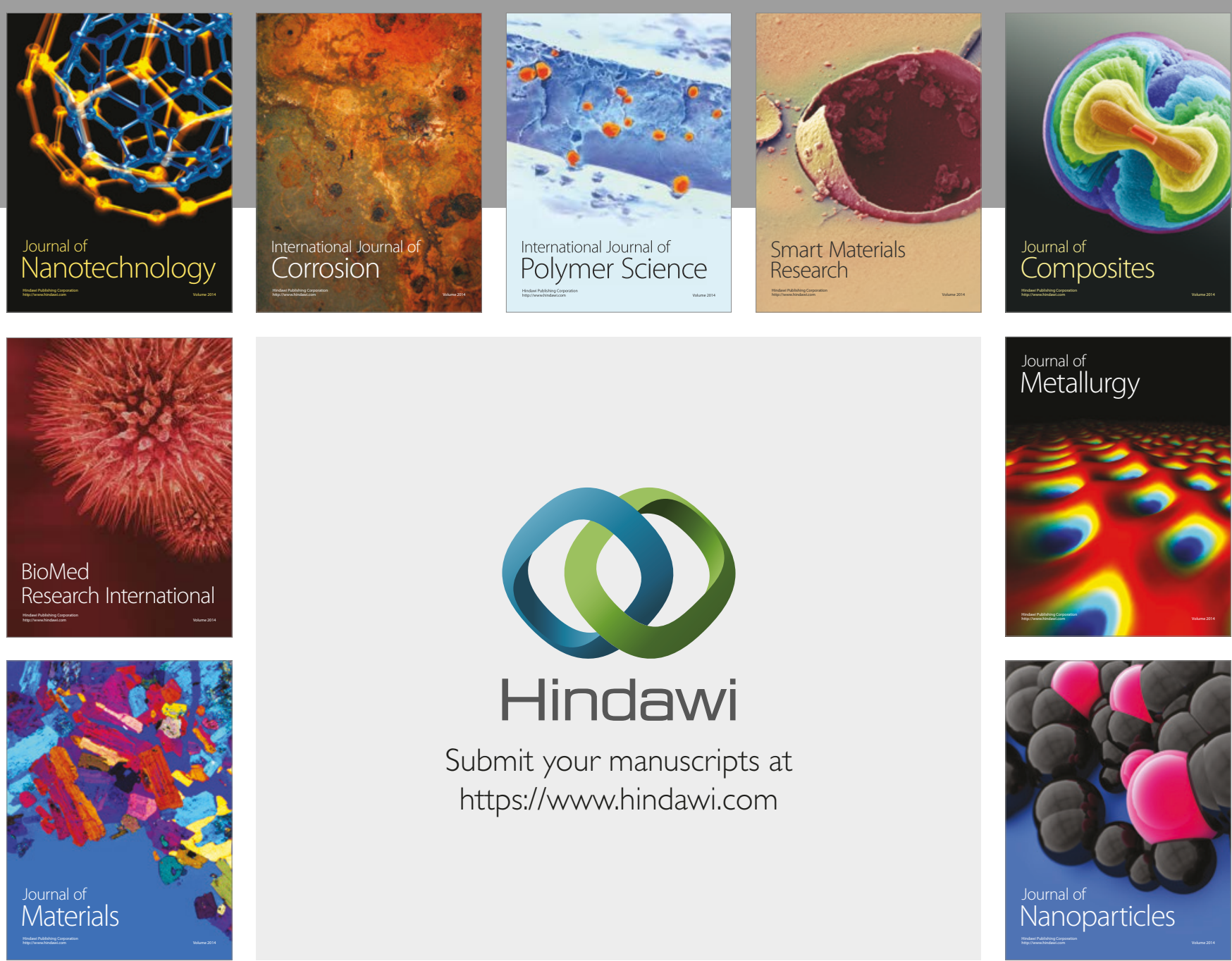

\section{Hindawi}

Submit your manuscripts at

https://www.hindawi.com
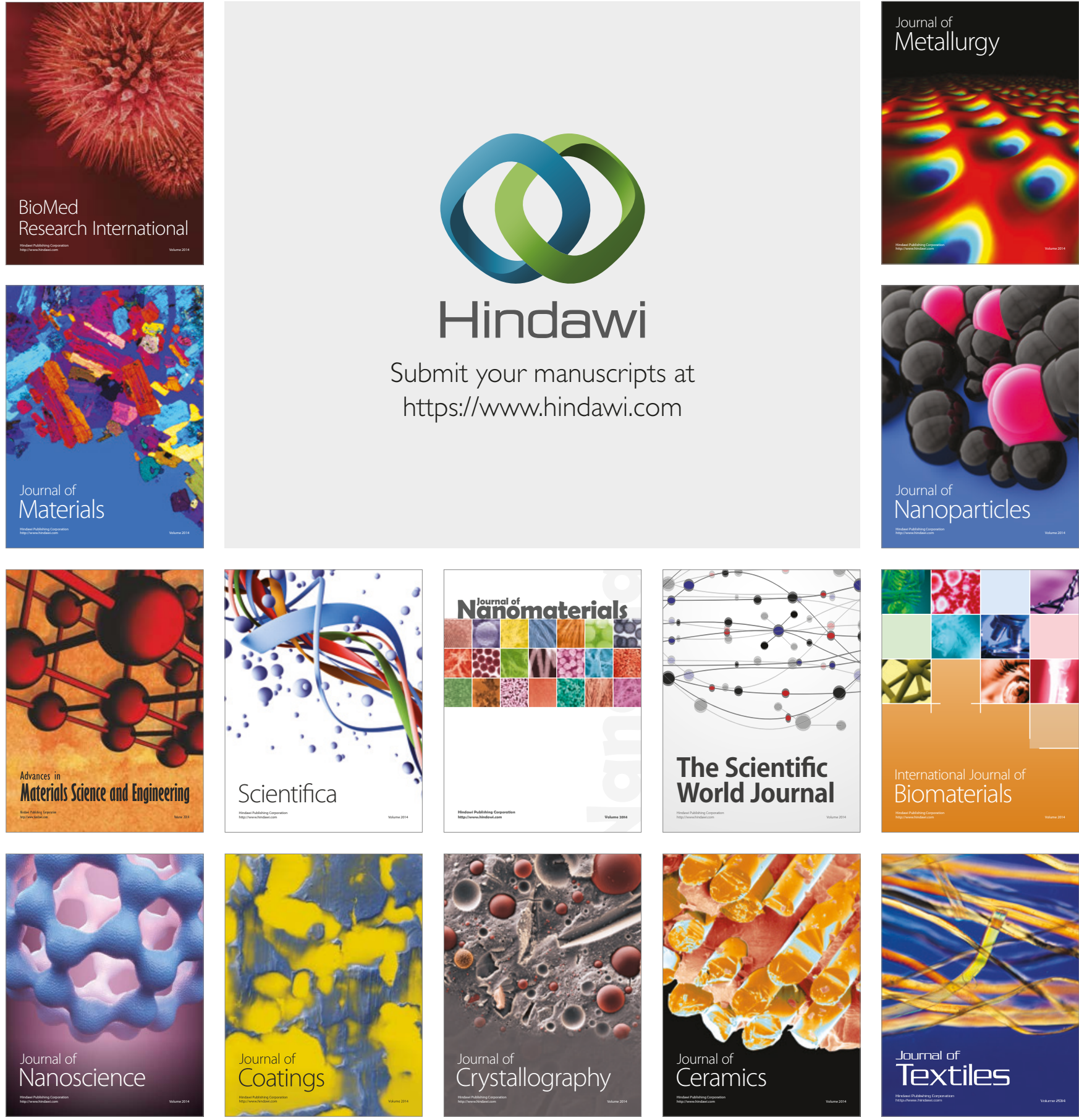

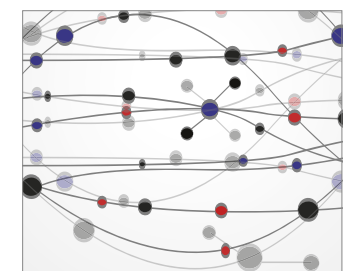

The Scientific World Journal
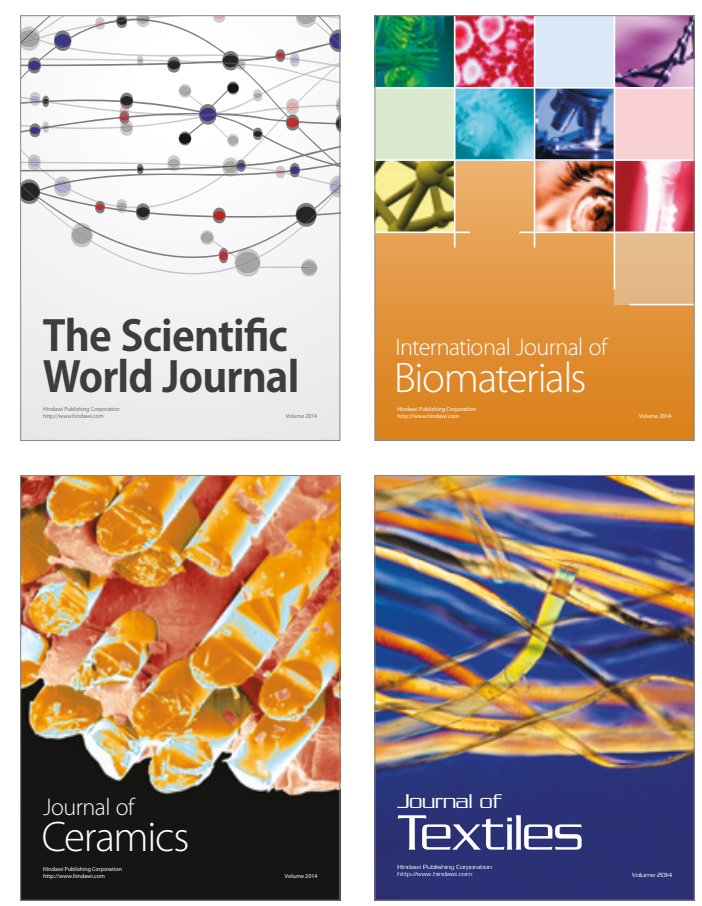